\title{
The Assessment of the Operational State of Traction Power Transformers by means of Decision Tables with Fuzzy Input Parameters
}

\author{
Vladimir A. Tremyasov and Vladislav G. Belov* \\ Siberian Federal University \\ Krasnoyarsk, Russian Federation
}

Received 24.02.2020, received in revised form 29.03.2020, accepted 21.05.2020

Abstract. The paper deals with the decision tables method in order to assess the state of traction substation transformers and to make control decisions. This approach allows us to evaluate the state of a substation power transformer with the changes in diagnostic parameters that were determined by means of a fuzzy set theory.

Keywords: traction substation, uncertainty of diagnostic parameters, decision tables, fuzzy set theory, membership function.

Citation: Tremyasov V.A., Belov V.G. The assessment of the operational state of traction power transformers by means of decision tables with fuzzy input parameters, J. Sib. Fed. Univ. Eng. \& Technol., 2020, 13(6), 721-731. DOI: 10.17516/1999494X-0261

\section{Оценка эксплуатационного состояния тяговых \\ силовых трансформаторов \\ с использованием таблиц решений \\ при нечетких входных параметрах}

В.А. Тремясов, В.Г. Белов

Сибирский федеральный университет Российская Федераџия, Красноярск

Аннотащия. В работе для оценки состояния трансформаторов тяговой подстанции и принятия управляющих решений предлагается метод с использованием таблиц решений. Применяя

(C) Siberian Federal University. All rights reserved

This work is licensed under a Creative Commons Attribution-Non Commercial 4.0 International License (CC BY-NC 4.0).

* Corresponding author E-mail address: belov_v2012@mail.ru 
этот подход, оценку состояния силового трансформатора подстанции можно дать с учетом изменений диагностических параметров, определенных на основе теории нечетких множеств.

Ключевые слова: тяговая подстанция, неопределенность диагностических параметров, таблицы решений, теория нечетких множеств, функция принадлежности.

Цитирование: Тремясов, В.А. Оценка эксплуатационного состояния тяговых силовых трансформаторов с использованием таблиц решений при нечетких входных параметрах / В.А. Тремясов, В.Г. Белов // Журн. Сиб. федер. ун-та. Техника и технологии, 2020. 13(6). С. 721-731. DOI: 10.17516/1999-494X-0261

\section{Введение}

Согласно стратегии развития ОАО «РЖД» до 2025 г., планируется произвести строительство 60 новых и техническое перевооружение существующих 359 тяговых подстанций (ТП) [1]. В связи с этим возросли требования к системе оценки текущего состояния электрооборудования (ЭО). Очередность технического обслуживания и ремонта (ТОиР) осуществляется путем оценивания текущего состояния электрооборудования ТП [2].

Состояния каждого энергообъекта (трансформаторов, воздушных линий, контактной сети, распределительных устройств) классифицируются таким образом: $N$ - не требуется вмешательства в эксплуатацию ЭО в предстоящий период; $A$ - требуется вмешательство без отключения оборудования в ближайшее время; В - требуется проведение ТОиР с отключением оборудования в течение предстоящего периода; $R$ - требуется вывод ЭО из работы для ТОиР.

Для всего парка энергообъектов составляют списки с оценками текущего состояния и разрабатывают графики ТОиР на квартал и на год.

Для принятия управляющих решений в процессе эксплуатации энергообъектов используют результаты мониторинга основных параметров ЭО, например данные хроматографического анализа трансформаторного масла.

Принимая во внимание неопределенность параметров и неполноту сведений об условиях эксплуатации конкретного ЭО, считаем обоснованным использовать таблицы решений с нечеткими входными параметрами [3-5]. Такой подход становится необходимым дополнением к таблицам решений, чтобы учесть неопределенность диагностических параметров в процессе эксплуатации энергообъектов тяговой подстанции.

\section{Метод оценки эксплуатационного состояния силового трансформатора}

Таблицы решений (ТР) получили большое распространение в исследовании операций, системном анализе и для математического обеспечения ЭВМ как удобное средство, позволяющее четко и просто описывать сложные ситуации. Описывая как саму задачу, так и необходимый для ее решения алгоритм, ТР в наглядной форме позволяют определять, какие условия должны быть выполнены, прежде чем можно будет переходить к тому или иному эксплуатационному действию [4]. Таблицы решений - способ компактного представления модели со сложной логикой. ТР в простой форме могут демонстрировать связь между множеством независимых условий и действий.

Применяются различные формы записи ТР. Рассмотрим табл. 1.

Строка (или группа) в табл. 1 является основным импликантом для текущего состояния энергообъекта. 
Таблица 1. Один из видов таблицы решений

Table 1. Main view of the solution table

\begin{tabular}{|c|c|c|c|c|c|}
\hline \multirow{2}{*}{ Строка } & \multicolumn{4}{|c|}{$\begin{array}{c}\text { Варианты условий } \\
\text { (диагностических параметров) }\end{array}$} & \multirow{2}{*}{$\begin{array}{c}\text { Возможные действия после классификации } \\
\text { состояния энергообъекта }\end{array}$} \\
\hline & $Y_{1}$ & $Y_{2}$ & $Y_{3}$ & $Y_{4}$ & \\
\hline 1 & $y_{11}$ & $y_{12}$ & $y_{13}$ & $y_{14}$ & $N$ \\
\hline 2 & $y_{21}$ & $y_{22}$ & $y_{23}$ & $y_{24}$ & $A$ \\
\hline 3 & $y_{31}$ & $y_{32}$ & $y_{33}$ & $y_{34}$ & $B$ \\
\hline 4 & $y_{41}$ & $y_{42}$ & $y_{43}$ & $y_{44}$ & $R$ \\
\hline
\end{tabular}

Теория нечетких множеств (ТНМ) представляет собой математический аппарат для работы с объектами, не имеющими жестких, однозначно задаваемых границ. Она позволяет формально описывать нестрогие, нечеткие, расплывчатые понятия и производить с ними различные операции. Применение ТНМ дает возможность формализовать процесс принятия решений в процессе эксплуатации при нечетких условиях [6-9].

Л. Заде ввел понятие лингвистической переменной [6], значениями которой являются слова или предложения естественного языка, которые описывают нечеткими значениями. Сегодня существуют достаточно четкие области, где классификация, а само собой и принимаемые решения, будут достаточно однозначными - область, близкая к идеальному состоянию ЭО, и область, близкая к полному износу ЭО [7]. Принимать решение сложней всего, когда состояние ЭО находится в переходном режиме между этими двумя крайними состояниями и когда этот переход не скачкообразен, а непрерывен. Такая ситуация типична для реально действующих систем, и многие понятия естественного языка не могут быть формализованы с помощью классических математических понятий, так как граница между двумя классифицируемыми состояниями (например, «новый» - «старый») является нечеткой, размытой [7].

Нечеткое подмножество $A$ множества $X$ характеризуется функцией принадлежности $\mathrm{m}$ : $X \rightarrow[0,1]$ ставит в соответствие каждому элементу $x \in X$ число $\mu_{\mathrm{A}}(x)$ из интервала $[0,1]$, характеризующее степень принадлежности элемента $x$ подмножеству $A$. Причем 0 и 1 представляют собой соответственно низшую и высшую степени принадлежности элемента к определенному подмножеству.

Таким образом, основное предположение состоит в том, что нечеткое множество, несмотря на расплывчатость его границ, может быть точно определено путем сопоставления каждому элементу $x$ числа, лежащего между 0 и 1 , которое представляет степень его принадлежности к $A$.

Над нечеткими множествами можно выполнять различные теоретико-множественные операции:

$$
\begin{aligned}
& \text { пересечения } C=A \cap B, \mu_{C}(x)=\mu_{A}(x) \& \mu_{B}(x) \text {; } \\
& \text { объединения } C=A \cup B, \mu_{C}(x)=\mu_{A}(x) \vee \mu_{B}(x) \text {; } \\
& \text { дополнения } C=\bar{A}, \mu_{C}(x)=1-\mu_{A}(x) .
\end{aligned}
$$

В работах $[10,11]$ был разработан метод так называемых треугольных нечетких чисел (ТНЧ), представляющих собой нечеткие числа с функцией принадлежности (ФП) в треугольном виде (рис. 1).

$$
-723-
$$




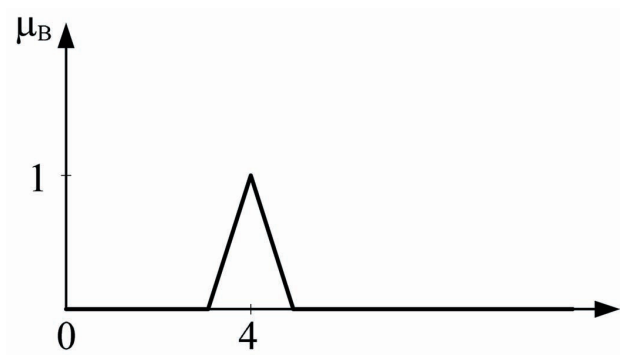

Рис. 1. Пример нечеткого множества. Пусть $B$-нечеткое число около 4

Fig. 1. Example of a fuzzy set. Let B be a fuzzy number about 4

Треугольное нечеткое число, которое часто обозначается $A=\left(a_{1}, a_{2}, a_{3}\right)$, определено следующей функцией принадлежности:

$$
\mu_{A}(x)= \begin{cases}\left(x-a_{1}\right) /\left(a_{2}-a_{1}\right) & \text { если } a_{1} \leq x \leq a_{2}, \\ \left(a_{3}-x\right) /\left(a_{3}-a_{2}\right) & \text { если } a_{2} \leq x \leq a_{3}, \\ 0 & \text { если } x \leq a_{1} \text { или } x \geq a_{3} .\end{cases}
$$

Вычисление $\alpha$-сечения $A_{\alpha}$ треугольного нечеткого числа выполняется по выражению

$$
A_{\alpha}=\left[a_{1}+\alpha\left(a_{2}-a_{1}\right) a_{3}-\alpha\left(a_{3}-a_{2}\right)\right]
$$

Каждый энергообъект получает оценки степени принадлежности к состояниям $N, \mathrm{~A}, \mathrm{~B}$, $R$ в соответствии с данными мониторинга диагностических параметров $\left\{\mathrm{y}_{i j}\right\}$. Для этого используют функции принадлежности, показанные на рис. 2. Такие функции строят для каждого диагностического параметра. Здесь: $Z$ - нулевой уровень, $L$ - низкий уровень, $\mathrm{M}-$ средний уровень, $\mathrm{H}$ - высокий уровень значения параметра. Если увеличение значения параметра $\mathrm{y}_{i j}$ свидетельствует о деградации состояния, то $Z$ отвечает нормальному состоянию $N, L$ - состоянию $\mathrm{A}, \mathrm{M}-\mathrm{B}, \mathrm{H}-R$. Если уменьшение значения параметра свидетельствует о деградации, тогда $Z$ отвечает состоянию $R, L$ - состоянию $\mathrm{B}, \mathrm{M}-\mathrm{A}, \mathrm{H}-N$. Значения функции принадлежности $\mu_{k}\left(\mathrm{y}_{i}\right)$ лежат в интервале $[0 ; 1]$. Функции имеют треугольную или линейную форму с перегибом (горизонтальный участок вне рабочего отрезка значений параметров).

Пример. Для оценки текущего состояния силового трансформатора была выбрана тяговая подстанция «Кача». В табл. 2 приведены значения диагностических параметров для тягового трансформатора ТП «Кача». Количество наблюдений равно $7(j=1, \ldots, 7)$, количество параметров $-7(i=1, \ldots, 7)$.

Критические и нормальные значения параметров взяты из справочной литературы [12] и приведены в табл. $3\left[y_{i}\right]_{k} k=8,9,10,11$ для абсолютно уверенного диагностирования $k$-го состояния объекта (при этих значениях параметров функция принадлежности имеет максимум $\left.\mu_{k}\left(y_{i}\right)=1\right)$.

Если произведены измерения и получен ряд значений $\left[\mathrm{y}_{i}\right] j, j=1, \ldots, 7$ для объекта или наблюдения $j$, причем, возможно, некоторые измерения отсутствуют, то ряд $\left[\mathrm{y}_{i}\right]_{k}$ образует таблицу оценок $\left[\mu_{X}\left(y_{i}\right)\right]_{k}$, а ряд $\left[y_{i}\right]_{j}-$ таблицу $\left[\mu_{X}\left(y_{i}\right)\right]_{j}$. Ниже приводятся две такие таблицы (табл. 4,5$)$ для рядов параметров и результатов наблюдений. 


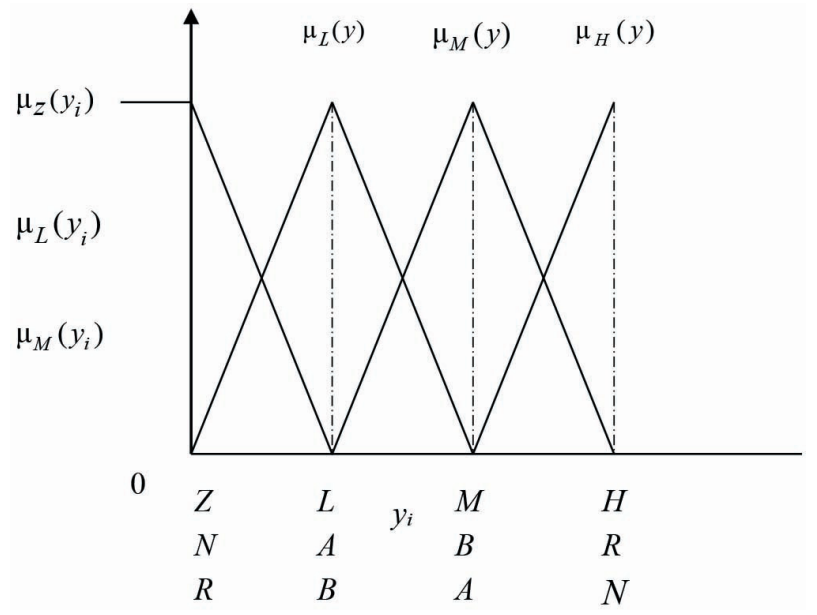

Рис. 2. Функции принадлежности диагностических параметров

Fig. 2. Functions of belonging of diagnostic parameters

Таблица 2. Результаты мониторинга параметров трансформаторного масла

Table 2. The monitoring results of parameters of transformer oil

\begin{tabular}{|c|c|c|c|c|c|c|c|c|}
\hline \multirow{2}{*}{$\begin{array}{c}\text { Номер } \\
\text { наблюдения }\end{array}$} & \multirow{2}{*}{$\begin{array}{c}\text { Дата } \\
\text { анализа }\end{array}$} & \multicolumn{7}{|c|}{ Значения входных параметров у } \\
\cline { 3 - 10 } & $\mathrm{CO}_{2}, \%$ & $\mathrm{CH}_{4}, \%$ & $\mathrm{C}_{2} \mathrm{H}_{6}, \%$ & $\mathrm{C}_{2} \mathrm{H}_{4}, \%$ & $\mathrm{C}_{2} \mathrm{H}_{2}, \%$ & $\mathrm{CO}, \%$ & $\operatorname{tg} \delta, \%$ \\
\hline 1 & 28.01 .19 & 0,098 & 0,0023 & 0,0012 & 0,009 & 0,0188 & 0,0137 & 0,15 \\
\hline 2 & 25.02 .19 & 0,107 & 0,0023 & 0,0012 & 0,0102 & 0,0194 & 0,0134 & 0,55 \\
\hline 3 & 04.03 .19 & 0,106 & 0,0024 & 0,0012 & 0,0102 & 0,0189 & 0,0146 & 1,15 \\
\hline 4 & 20.05 .19 & 0,135 & 0,0016 & 0,0011 & 0,0095 & 0,0175 & 0,0143 & 0,68 \\
\hline 5 & 20.06 .19 & 0,156 & 0,0013 & 0,0010 & 0,0083 & 0,0143 & 0,0193 & 0,88 \\
\hline 6 & 19.07 .19 & 0,150 & 0,0012 & 0,0009 & 0,0076 & 0,0125 & 0,0140 & 0,30 \\
\hline 7 & 20.09 .19 & 0,156 & 0,0009 & 0,0008 & 0,0069 & 0,0101 & 0,0159 & 0,53 \\
\hline
\end{tabular}

Таблица 3. Ряды критических значений эксплуатационных параметров трансформаторного масла

Table 3. Series of critical values of operational parameters of transformer oil

\begin{tabular}{|c|c|c|c|c|c|c|c|}
\hline \multirow{3}{*}{ 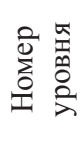 } & \multicolumn{7}{|c|}{ Критические и нормальные значения параметров у } \\
\hline & $\mathrm{CO}_{2}, \%$ & $\mathrm{CH}_{4}, \%$ & $\mathrm{C}_{2} \mathrm{H}_{6}, \%$ & $\mathrm{C}_{2} \mathrm{H}_{4}, \%$ & $\mathrm{C}_{2} \mathrm{H}_{2}, \%$ & $\mathrm{CO}, \%$ & $\operatorname{tg} \delta, \%$ \\
\hline & 1 & 2 & 3 & 4 & 5 & 6 & 7 \\
\hline \multirow{2}{*}{8} & \multicolumn{7}{|c|}{ Критические уровни для состояния $B$ (необходима смена масла) } \\
\hline & 0,07 & 0,003 & 0,003 & 0,001 & 0,0002 & 0,03 & 3,0 \\
\hline \multirow{2}{*}{9} & \multicolumn{7}{|c|}{ Критические уровни для состояния $R$ (необходим ремонт) } \\
\hline & 0,2 & 0,01 & 0,005 & 0,01 & 0,01 & $0,05-0,06$ & 5,0 \\
\hline \multirow{2}{*}{10} & \multicolumn{7}{|c|}{ Критические уровни для состояния $A$ (необходимо внимание) } \\
\hline & $0,05-0,06$ & 0,001 & 0,001 & 0,005 & 0,0001 & 0,015 & 1,0 \\
\hline \multirow{2}{*}{11} & \multicolumn{7}{|c|}{ Нормальные уровни параметров (состояние $N$ ) } \\
\hline & 0,01 & 0,00013 & 0,0001 & 0,0002 & 0,00005 & 0,007 & 0,05 \\
\hline
\end{tabular}


Таблица 4. Результаты наблюдений параметров $\left[\mu_{X}\left(y_{i}\right)\right]_{1}$

Table 4 . The results of observations $\left[\mu_{X}\left(y_{i}\right)\right]_{1}$

\begin{tabular}{|c|c|c|c|c|c|c|c|}
\hline$i$ & 1 & 2 & 3 & 4 & 5 & 6 & 7 \\
\hline$H$ & 0 & 0,4 & 0 & 0 & 0 & 0 & 0,857 \\
\hline$M$ & 0 & 0 & 1 & 0 & 0 & 0,857 & 0,14 \\
\hline$L$ & 0,14 & 0 & 0 & 0,7 & 0 & 0,14 & 0 \\
\hline$Z$ & 0,857 & 0,57 & 0 & 0,28 & 1 & 0 & 0 \\
\hline
\end{tabular}

Таблица 5. Нормальные уровни параметров $\left[\mu_{X}\left(y_{i}\right)\right]_{11}$

Table 5. The results of observations $\left[\mu_{X}\left(y_{i}\right)\right]_{11}$

\begin{tabular}{|l|l|l|l|l|l|l|l|}
\hline$i$ & 1 & 2 & 3 & 4 & 5 & 6 & 7 \\
\hline$H$ & 0 & 0 & 0 & 0 & 0 & 0 & 0 \\
\hline$M$ & 0 & 0 & 0 & 0 & 0 & 0 & 0 \\
\hline$L$ & 0 & 0 & 0 & 0 & 0 & 0 & 0 \\
\hline$Z$ & 1 & 1 & 1 & 1 & 1 & 1 & 1 \\
\hline
\end{tabular}

Таблица 6. Таблица решений для состояния $N$

Table 6. Solution table for state $N$

\begin{tabular}{|c|c|c|c|c|c|c|c|c|c|}
\hline \multirow{2}{*}{$\begin{array}{c}\text { Состояния трансформатора } \\
\text { при уровнях параметров }\end{array}$} & \multicolumn{6}{|c|}{ Функции принадлежности параметров $\mu_{X}\left(y_{i}\right)$} & \multirow{2}{*}{$\begin{array}{c}\text { Выход } \\
\mu_{X}\left(y_{i}\right)_{\mathrm{cp}}\end{array}$} \\
\cline { 2 - 9 } & 1 & 2 & 3 & 4 & 5 & 6 & 7 & 0 \\
\hline$R(Z)$ & 0 & 0 & 0 & 0 & 0 & 0 & 0 & 0 \\
\hline$B(L)$ & 0 & 0 & 0 & 0 & 0 & 0 & 0 & 0 \\
\hline$A(M)$ & 0 & 0 & 0 & 0 & 0 & 0 & 0 & 0 \\
\hline$N(H)$ & 0 & 0,57 & 0 & 0 & 0 & 0 & 0,857 & 0,204 \\
\hline
\end{tabular}

Накладывая таблицу $\left[\mu_{X}\left(y_{i}\right)\right]_{1}$ на $\left[\mu_{X}\left(y_{i}\right)\right]_{11}$, отмечаем совпадение ненулевых элементов. Чем больше число совпадений, тем больше уверенность в том, что объект близок к состоянию 11, т.е. к $N$. Необходимо оценить степень уверенности. Для этого вычисляем в каждой клетке совмещенной таблицы степень принадлежности для пересечения размытых множеств $X_{j}$ и $X_{k}$, которая определяется как минимум из $\mu_{X}\left(y_{i}\right)_{j}$ и $\mu_{X}\left(y_{i}\right)_{k}$, и строим таблицу $\left[\mu_{X}\left(y_{i}\right)\right]_{j k}$ :

$$
\mu_{X}\left(y_{j}\right)_{j k}=\min \left(\mu_{X}\left(y_{i}\right)_{j}, \mu_{X}\left(y_{i}\right)_{k}\right) .
$$

Например, наложив приведенную выше таблицу $\left[\mu_{X}\left(y_{i}\right)\right]_{1}$ на $\left[\mu_{X}\left(y_{i}\right)\right]_{11}$, получим таблицу решений $\left[\mu_{X}\left(y_{i}\right)\right]_{1,11}$ следующего вида (табл. 6).

При этом наложении отмечаем две точки совпадения лингвистических оценок. Мерой совпадения в каждой точке является степень принадлежности и $\mathrm{y}_{i j}, \mathrm{y}_{i k}$ в этой точке к одному 
и тому же множеству (любому из множеств $\mathrm{H}, \mathrm{M}, L, Z$ ). Это значит, что эта мера представляет собой меру принадлежности к объединению нечетких множеств в каждой точке. Функция принадлежности к объединению в каждой точке совпадения $i$ определяется как

$$
\mu_{X}\left(y_{i}\right)=\max _{Y, X}\left[\left(\mu_{X}\left(y_{i}\right), \mu_{Y}\left(y_{i}\right)\right)\right]_{i},
$$

здесь $Y$ и $X$-лингвистические переменные для точки $i$; $\mu_{X}\left(y_{i}\right)$ - мера совпадения значений параметра $i$ (результата измерения или наблюдения) и критического значения (признака).

Для нашего примера значения $\mu_{X}\left(y_{i}\right)$ составят: $0 ; 0,57 ; 0 ; 0 ; 0 ; 0 ; 0,857$; что в среднем дает $\mu_{X}\left(y_{i}\right)_{\mathrm{p}}=[0,57+0,857] / 7=0,204$. Это означает, что уверенность заключения о возможности состояния $N$ объекта при наблюдении № 1 составляет около 20,4 \% при семи критериях за выбранный период времени.

Аналогичным образом можно оценить возможности остальных состояний по всем наблюдениям. Значения функции принадлежности в соответствии с линейным характером ее изменения на отрезке между критическими значениями параметра вычисляют по следующим формулам:

$$
\begin{aligned}
& y_{i}(X) \leq y_{i j} \leq y_{i}(Y), \text { то } \mu_{Y}\left(y_{i}\right)_{j}=\left[y_{i j}-y_{i}(X)\right] /\left[y_{i}(Y)-y_{i}(X)\right] ; \\
& \mu_{X}\left(y_{i}\right)_{j}=1-\mu_{Y}\left(y_{i}\right)_{j} ; X(Y) \in N, A, B, R .
\end{aligned}
$$

Когда наблюдения относятся к парку трансформаторов, то можно с помощью оценок уверенности сформировать списки и установить очередность обслуживания и ремонта. Если наблюдения относятся к различным моментам (датам) «жизни» одного трансформатора, то можно построить графики изменения уверенности в классификации состояний (рис. 3-6) и по ним прогнозировать остаточный ресурс, задав критические значения уверенности в необходимости производства ТОиР.

Таким образом, за отчетный период наблюдений для состояний $A$, В и $R$ будут соответствовать следующие таблицы решений (табл. 7 - 9).

По приведенным графикам изменения уверенности состояний (рис. 3-6) можно утверждать, в какие периоды и в каком состоянии находились измеряемые параметры, превышали они допустимую норму или же нет, в какой момент состояние стабилизировалось. Например, рассматривая график уверенности состояния $N$ (рис. 3), можно сказать, что за отчетный

Таблица 7. Таблица решений для состояния $A$

Table 7. Solution table for state $A$

\begin{tabular}{|c|c|c|c|c|c|c|c|c|}
\hline $\begin{array}{c}\text { Состояния трансформатора } \\
\text { при уровнях параметров }\end{array}$ & \multicolumn{7}{|c|}{ Функции принадлежности параметров $\mu_{X}\left(y_{i}\right)$} & $\begin{array}{c}\text { Выход } \\
\mu_{X}\left(y_{i}\right)_{\mathrm{cp}}\end{array}$ \\
\hline & 1 & 2 & 3 & 4 & 5 & 6 & 7 & 0 \\
\hline$R(Z)$ & 0 & 0 & 0 & 0 & 0 & 0 & 0 & 0 \\
\hline$B(L)$ & 0 & 0 & 0 & 0 & 0 & 0 & 0 & 0 \\
\hline$A(M)$ & 0 & 0 & 1 & 0 & 0 & 0,857 & 0 & 0,265 \\
\hline$N(H)$ & 0 & 0 & 0 & 0 & 0 & 0 & 0 & 0 \\
\hline
\end{tabular}


Таблица 8. Таблица решений для состояния $B$

Table 8 . Solution table for state $B$

\begin{tabular}{|c|c|c|c|c|c|c|c|c|}
\hline \multirow{2}{*}{$\begin{array}{c}\text { Состояния трансформатора } \\
\text { при уровнях параметров }\end{array}$} & \multicolumn{6}{|c|}{ Функции принадлежности параметров $\mu_{X}\left(y_{i}\right)$} & \multirow{2}{*}{$\begin{array}{c}\text { Выход } \\
\mu_{X}\left(y_{i}\right)_{\mathrm{cp}}\end{array}$} \\
\cline { 2 - 10 }$n$ & 1 & 2 & 3 & 4 & 5 & 6 & 7 & 0 \\
\hline$R(Z)$ & 0 & 0 & 0 & 0 & 0 & 0 & 0 & 0,220 \\
\hline$B(L)$ & 0,14 & 0,56 & 0 & 0,7 & 0 & 0,14 & 0 & 0 \\
\hline$A(M)$ & 0 & 0 & 0 & 0 & 0 & 0 & 0 & 0 \\
\hline$N(H)$ & 0 & 0 & 0 & 0 & 0 & 0 & 0 & 0 \\
\hline
\end{tabular}

Таблица 9. Таблица решений для состояния $R$

Table 9. Solution table for state $R$

\begin{tabular}{|c|c|c|c|c|c|c|c|c|}
\hline \multirow{2}{*}{$\begin{array}{c}\text { Состояния трансформатора } \\
\text { при уровнях параметров }\end{array}$} & \multicolumn{7}{|c|}{ Функции принадлежности параметров $\mu_{X}\left(y_{i}\right)$} & $\begin{array}{c}\text { Выход } \\
\mu_{X}\left(y_{i}\right)_{\mathrm{cp}}\end{array}$ \\
\cline { 2 - 9 }$n$ & 1 & 2 & 3 & 4 & 5 & 6 & 7 & 0,305 \\
\hline$R(Z)$ & 0,857 & 0 & 0 & 0,28 & 1 & 0 & 0 & 0 \\
\hline$B(L)$ & 0 & 0 & 0 & 0 & 0 & 0 & 0 & 0 \\
\hline$A(M)$ & 0 & 0 & 0 & 0 & 0 & 0 & 0 & 0 \\
\hline$N(H)$ & 0 & 0 & 0 & 0 & 0 & 0 & 0 & 0 \\
\hline
\end{tabular}

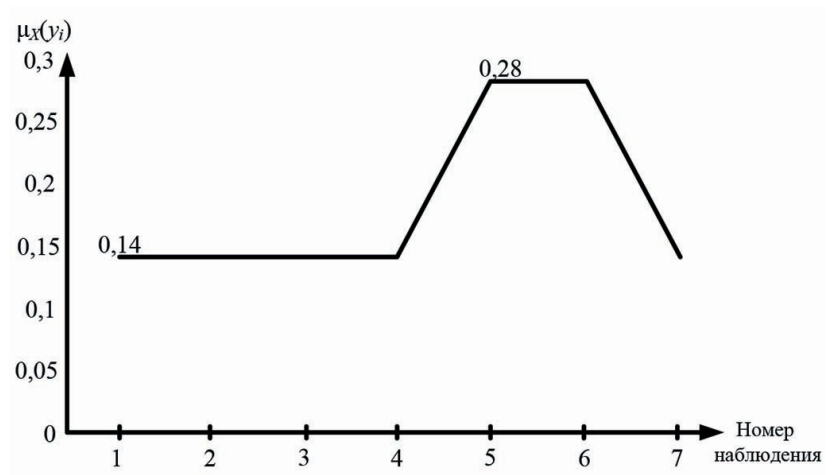

Рис. 3. График изменения уверенности состояния $N$

Fig. 3. Graph of changes in the confidence state $N$

период не были превышены критические значения уверенности для данного состояния оборудования $[2,12]$ и нет необходимости в проведении ТОиР. Если значения параметров превышают «норму», т.е. величины, которые можно ожидать за счет естественного старения, то через некоторое время (5- 10 дней) повторно отбирают пробу масла и проводят анализ. В случае превышения критических значений следует ТОиР. Из полученных таблиц (табл. 6-9) для нашего примера, при наблюдении семи критериев за выбранный период времени, для каждого состояния приводится возможная уверенность его существования. Так, для состояния $N$ она составляет около $20,4 \%$, для состояния $A-26,5 \%$, для состояния $B-22,2 \%$, для состояния $R-30,5 \%$. 


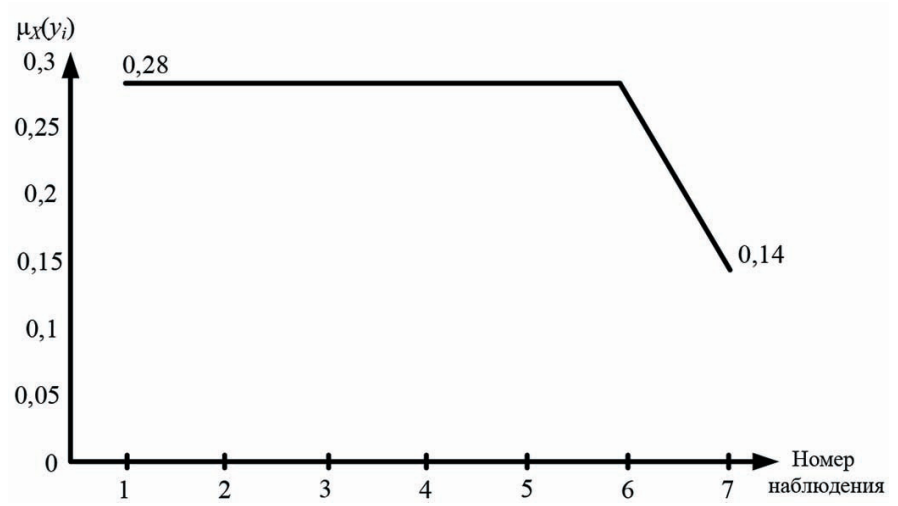

Рис. 4. График изменения уверенности состояния $A$

Fig. 4. Graph of changes in the confidence state $A$

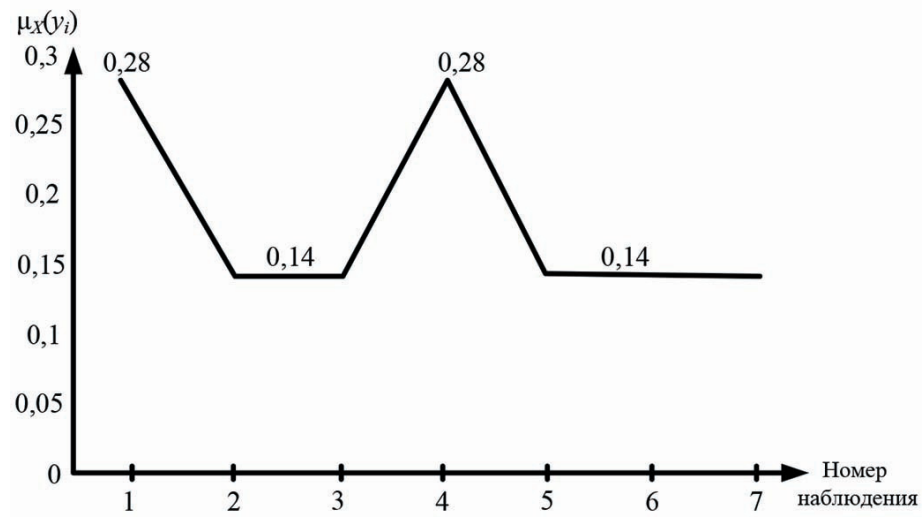

Рис. 5. График изменения уверенности состояния $B$

Fig. 5. Graph of changes in the confidence state $B$

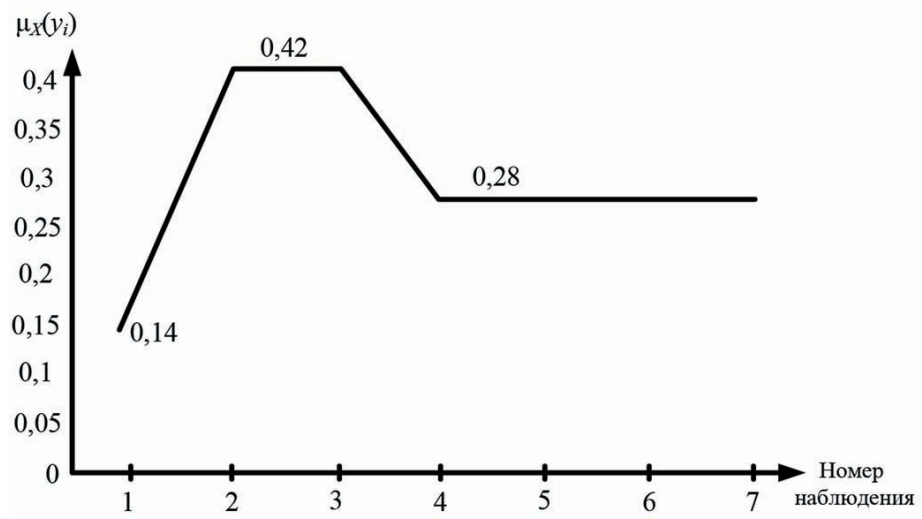

Рис. 6. График изменения уверенности состояния $R$

Fig. 6. Graph of changes in the confidence state $R$ 


\section{Заключение}

Рассмотренный выше метод оценивания состояния ЭО может быть положен в основу программного обеспечения экспертной системы для службы эксплуатации высоковольтного оборудования тяговых подстанций железнодорожного транспорта. Информация о результатах текущих испытаний и измерений параметров ЭО регулярно вводится и обрабатывается на компьютерах работниками службы эксплуатации. Инженеры по ЭО ТП, воздушным линиям и контактной сети получают оценки уверенности в идентификации состояния каждого поднадзорного энергообъекта по мере поступления новых сведений. На основе этих оценок составляют и корректируют план текущих и капитальных ремонтов.

Предполагается, что такая экспертная система позволит сократить трудовые и материальные затраты на ТОиР и обеспечить надежность старого оборудования.

\section{Список литературы / References}

[1] Распоряжение Правительства РФ от 17.06.2008 N 877-р О Стратегии развития железнодорожного транспорта в Российской Федерации до 2030 года [Order of the Government of the Russian Federation of June 17, 2008 N 877-r On the Strategy for the Development of Railway Transport in the Russian Federation until 2030 (in Russian)]

[2] Васильев А.П., Гук Ю.Б., Карпов В.В. Надежность электроэнергетических установок и систем. Теория и практика. СПб.: ГУ «Ленгосэнергонадзор», 2000 [Vasiliev A.P., Guk Y.B., Karpov V.V. Reliability of electric power installations and systems. Theory and practice. SPb.: GU "Langselector", 2000 (in Russian)]

[3] Pollack S.L. Decision Tables: Theory and Practice. New-York, Wiley-Interscience, 1971.

[4] Хамби Э. Программирование таблиц решений, пер. с англ.; под ред. Э.З. Любимского. М.: Мир, 1976. С. 88 [Khambi E. Programming tables of solutions, translation from English; ed. by E.Z. Lyubimsky. M.: Mir, 1976. P. 88 (in Russian)]

[5] Тремясов В.А. Анализ надежности электроустановок на основе таблиц решений Оптимизация режимов электропотребления промышленных предприятий и районов. Межвуз. сб. Красноярск: КрПИ, 1990, с. 43-46. [Tremasov, V.A. Analysis of reliability of electrical installation sonth ebasis of decisiontables. Optimization of power consumption modes of industrial enterprises and districts. Krasnoyarsk: KrPI, 1990, p. 43-46 (in Russian)]

[6] Zadeh L.A. Fuzzy sets as a basis for theory of possibility. Fuzzy Sets and Systems, 1978, (1), 3-28.

[7] Горшкова К.Л. Применение нечеткой логики и нечетких регуляторов в АСУТП. Ученые записки Альметьевского государственного нефтяного института, 2011, 180-184. [Gorshkova K.L. Application of fuzzy logic and fuzzy controllers in the automated control system. Scientific notes of the Almetyevsk state oil Institute, 2011, 180-184 (in Russian)]

[8] Орловский С.А. Проблемы принятия решений при нечеткой исходной информации. М.: Наука. Главная редакция физико-математической литературы, 1981. 208 c. [Orlovsky S.A. Decision making problems with fuzzy initial information. M.: Science. The main edition of the physical and mathematical literature, 1981, 208 p. (in Russian)]

[9] Ягер Р.Р. Нечеткие множества и теория возможностей. Последние достижения. М.: Радио и связь, 1986, 405 с. [Jager R.R. Fuzzy sets and the theory of possibilities. Recent achievements. Moscow: Radio and communications, 1986, 405 p. (in Russian)] 
[10] Li W., Xiong X., Zhou J., Fuzzy models of overhead power line weather-related outages. IEEE Trans. Power Sys. Aug. 2008, 23 (3), 1529-1531.

[11] Li W., Xiong X., Zhou J. Incorporating fuzzy models weather-related outages in transmission system relability assesment. IETProceed. Generation, Transmiss. Distribut. Jan. 2009, 3(1), 26-37.

[12] ВСН 359-76/ММСССССР Нормы технологического проектирования тяговых сетей и подстанций для промышленного железнодорожного транспорта нормальной колеи, в том числе для Северной строительно-климатической зоны. [VSN 359-76 / MMSS of the USSR Standards of technological design of traction networks and substations for industrial railway transport of normal gauge, including for the Northern construction and climate zone (in Russian)] 